See Mun Lee, Kong Mun Lo and Edward R.T. Tiekink*

\title{
Crystal structure of aqua-2,2',2"-(((nitrilo- $\mathrm{k} N$-tris(ethane-2,1-diyl))tris (azanylylidene- $\left.\mathrm{K}^{3} \mathrm{~N}^{\prime}, N^{\prime \prime}, N^{\prime \prime \prime}\right)$ )tris(methanylylidene))tris (4-chlorophenolato- $\mathrm{K}^{3} \mathrm{O}, \mathrm{O}^{\prime}, \mathrm{O}^{\prime \prime}$ ) neodymium(III), $\mathrm{C}_{27} \mathrm{H}_{26} \mathrm{Cl}_{3} \mathrm{~N}_{4} \mathrm{NdO}_{4}$
}

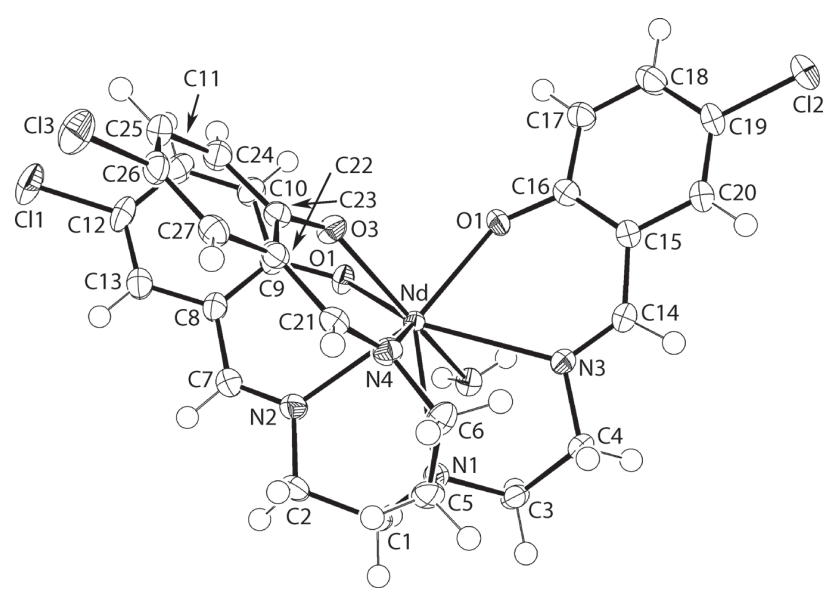

https://doi.org/10.1515/ncrs-2019-0095

Received January 29, 2019; accepted March 11, 2019; available online March 30, 2019

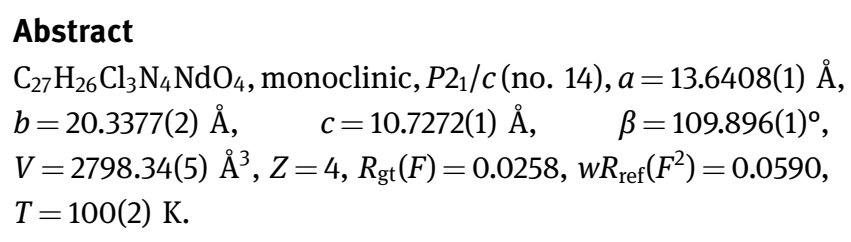

CCDC no.: 1902338

The molecular structure is shown in the figure. Table 1 contains crystallographic data and Table 2 contains the list of the atoms including atomic coordinates and displacement parameters.

\section{Source of material}

The ligand, tris\{[(5-chlorosalicylidene)amino]ethyl $\}$ amine was prepared from a 1:3 molar ratio of 5-chlorosalicylaldehyde (Sigma-Aldrich) and tris(2-aminoethyl)amine (Sigma-Aldrich)

\footnotetext{
*Corresponding author: Edward R.T. Tiekink, Research Centre for Crystalline Materials, School of Science and Technology, Sunway University, 47500 Bandar Sunway, Selangor Darul Ehsan, Malaysia, e-mail: edwardt@sunway.edu.my,Edward.Tiekink@gmail.com. https://orcid.org/0000-0003-1401-1520

See Mun Lee and Kong Mun Lo: Research Centre for Crystalline Materials, School of Science and Technology, Sunway University, 47500 Bandar Sunway, Selangor Darul Ehsan, Malaysia
}

Table 1: Data collection and handling.

\begin{tabular}{ll}
\hline Crystal: & Purple prism \\
Size: & $0.16 \times 0.12 \times 0.05 \mathrm{~mm}$ \\
Wavelength: & Mo $K \alpha$ radiation $(0.71073 \AA$ A $)$ \\
$\mu:$ & $2.18 \mathrm{~mm}^{-1}$ \\
Diffractometer, scan mode: & $\mathrm{CCD}, \varphi$ and $\omega$ \\
$\theta_{\text {max }}$, completeness: & $30.5^{\circ}, 99 \%$ \\
$N(h k l)_{\text {measured }}, N(h k l)_{\text {unique }}, R_{\text {int }}:$ & $25994,7981,0.026$ \\
Criterion for $I_{\text {obs }}, N(h k l)_{\mathrm{gt}}:$ & $I_{\text {obs }}>2 \sigma\left(I_{\text {obs }}\right), 6843$ \\
$N(\text { param })_{\text {refined }}:$ & 358 \\
Programs: & Bruker [1], SHELX [2-4], \\
& WinGX/ORTEP [5]
\end{tabular}

in methanolic solution [6]. Both the ligand (0.56 g, $1 \mathrm{mmol})$ and triethylamine $(0.14 \mathrm{~mL}, 1.0 \mathrm{mmol})$ were dissolved in absolute ethanol $(25 \mathrm{~mL})$ and refluxed for $1 \mathrm{~h}$. After that, an ethanolic solution (15 mL) of neodymium(III) nitrate hexahydrate (SigmaAldrich; $0.44 \mathrm{~g}, 1 \mathrm{mmol}$ ) was added to the mixture which was further refluxed for $3 \mathrm{~h}$ and filtered. The filtrate was evaporated until a precipitate was obtained. The precipitate was recrystallised from ethanol solution and the by-product, triethylammonium chloride, was removed through filtration. Purple needles of the title compound (alternative name: aqua(tris\{2-[(5-chloro-2-oxidobenzylidene$\kappa O$ )amino- $\kappa N$ ]ethyl\}amine- $\kappa N$ )neodymium(III) suitable for $\mathrm{X}$-ray crystallographic studies were obtained from the slow evaporation of the filtrate. Yield: $0.36 \mathrm{~g}(50 \%)$. M.pt: 430-434 K. IR ( $\left.\mathrm{cm}^{-1}\right) 1625(\mathrm{~s}) v(\mathrm{C}=\mathrm{N}), 1526(\mathrm{~m}), 1462(\mathrm{~m})$, $1390(\mathrm{~m}) v(-\mathrm{O}-\mathrm{C}=\mathrm{C}-), 1160(\mathrm{~m}) v(\mathrm{C}-\mathrm{O}-\mathrm{C})$.

\section{Experimental details}

The C-bound $\mathrm{H}$ atoms were geometrically placed $(\mathrm{C}-\mathrm{H}=0.95-0.99 \AA)$ and refined as riding with $U_{\text {iso }}(\mathrm{H})=1.2 U_{\text {eq }}(\mathrm{C})$. The O-bound $\mathrm{H}$-atoms were located in difference Fourier maps but were refined with a distance restraint of $\mathrm{O}-\mathrm{H}=0.84+-0.01 \AA$, and with $U_{\text {iso }}(\mathrm{H})$ set to $1.5 U_{\text {equiv }}(0)$. Owing to poor agreement, two reflections, i.e. (1 00 ) and (14 17 3), were removed from the final cycles of refinement. The maximum and minimum electron density peaks of 1.13 and 0.78 e $\AA^{-3}$, respectively, were located 0.80 and $1.33 \AA$ from the $\mathrm{Nd}$ and $\mathrm{H} 17$ atoms, respectively. 
Table 2: Fractional atomic coordinates and isotropic or equivalent isotropic displacement parameters $\left(\AA^{2}\right)$.

\begin{tabular}{|c|c|c|c|c|}
\hline Atom & $x$ & $y$ & $z$ & $U_{\text {iso }} * / U_{\text {eq }}$ \\
\hline $\mathrm{Nd}$ & $0.05622(2)$ & $0.61064(2)$ & $0.40249(2)$ & $0.01016(3)$ \\
\hline $\mathrm{Cl} 1$ & $0.60937(4)$ & $0.56005(3)$ & $0.83944(6)$ & $0.02975(14)$ \\
\hline $\mathrm{Cl} 2$ & $-0.42199(5)$ & $0.71835(4)$ & $0.60792(6)$ & $0.03063(14)$ \\
\hline $\mathrm{Cl} 3$ & $0.47697(5)$ & $0.88849(3)$ & $0.67008(6)$ & $0.02568(12)$ \\
\hline 01 & $0.16097(11)$ & $0.53052(7)$ & $0.55077(14)$ & $0.0146(3)$ \\
\hline 02 & $-0.04437(11)$ & $0.61118(7)$ & $0.53981(14)$ & $0.0138(3)$ \\
\hline 03 & $0.17656(11)$ & $0.67202(8)$ & $0.56006(14)$ & $0.0155(3)$ \\
\hline $04 \mathrm{~W}$ & $-0.02914(12)$ & $0.50248(8)$ & $0.32709(14)$ & $0.0146(3)$ \\
\hline $\mathrm{H} 1 \mathrm{~W}$ & $-0.0784(15)$ & $0.4987(14)$ & $0.356(2)$ & $0.022^{\star}$ \\
\hline $\mathrm{H} 2 \mathrm{~W}$ & $0.0079(18)$ & $0.4703(9)$ & $0.361(2)$ & $0.022^{*}$ \\
\hline $\mathrm{N} 1$ & $0.00753(13)$ & $0.61455(9)$ & $0.13345(17)$ & $0.0136(3)$ \\
\hline $\mathrm{N} 2$ & $0.20733(14)$ & $0.57302(10)$ & $0.32562(17)$ & $0.0163(4)$ \\
\hline N3 & $-0.14468(13)$ & $0.63584(9)$ & $0.26254(17)$ & $0.0140(3)$ \\
\hline N4 & $0.08638(14)$ & $0.72383(9)$ & $0.30762(17)$ & $0.0143(3)$ \\
\hline $\mathrm{C} 1$ & $0.07921(17)$ & $0.56754(12)$ & $0.1020(2)$ & $0.0182(4)$ \\
\hline $\mathrm{H} 1 \mathrm{~A}$ & 0.070210 & 0.570987 & 0.006652 & $0.022^{*}$ \\
\hline H1B & 0.060774 & 0.522165 & 0.119093 & $0.022^{\star}$ \\
\hline $\mathrm{C} 2$ & $0.19230(17)$ & $0.58064(13)$ & $0.1837(2)$ & $0.0214(5)$ \\
\hline $\mathrm{H} 2 \mathrm{~A}$ & 0.237834 & 0.549390 & 0.158250 & $0.026^{\star}$ \\
\hline $\mathrm{H} 2 \mathrm{~B}$ & 0.211592 & 0.625807 & 0.166528 & $0.026^{*}$ \\
\hline $\mathrm{C} 3$ & $-0.10032(16)$ & $0.58985(11)$ & $0.0754(2)$ & $0.0150(4)$ \\
\hline $\mathrm{H} 3 \mathrm{~A}$ & -0.103058 & 0.543818 & 0.104023 & $0.018^{*}$ \\
\hline H3B & -0.120921 & 0.590099 & -0.022481 & $0.018^{\star}$ \\
\hline $\mathrm{C} 4$ & $-0.17747(16)$ & $0.63102(12)$ & $0.1162(2)$ & $0.0159(4)$ \\
\hline $\mathrm{H} 4 \mathrm{~A}$ & -0.181854 & 0.675632 & 0.077803 & $0.019^{\star}$ \\
\hline H4B & -0.247515 & 0.610721 & 0.081206 & $0.019^{*}$ \\
\hline $\mathrm{C} 5$ & $0.01757(17)$ & $0.68092(11)$ & $0.0815(2)$ & $0.0168(4)$ \\
\hline $\mathrm{H} 5 \mathrm{~A}$ & -0.038607 & 0.687042 & -0.005026 & $0.020^{*}$ \\
\hline $\mathrm{H} 5 \mathrm{~B}$ & 0.085101 & 0.683811 & 0.066363 & $0.020^{*}$ \\
\hline $\mathrm{C} 6$ & $0.01154(17)$ & $0.73578(11)$ & $0.1743(2)$ & $0.0169(4)$ \\
\hline $\mathrm{H} 6 \mathrm{~A}$ & 0.027280 & 0.778355 & 0.140489 & $0.020^{\star}$ \\
\hline H6B & -0.059892 & 0.738134 & 0.178029 & $0.020^{*}$ \\
\hline $\mathrm{C} 7$ & $0.29957(17)$ & $0.55877(12)$ & $0.4017(2)$ & $0.0181(4)$ \\
\hline $\mathrm{H} 7$ & 0.351231 & 0.553503 & 0.361412 & $0.022^{\star}$ \\
\hline $\mathrm{C} 8$ & $0.33250(16)$ & $0.54988(11)$ & $0.5454(2)$ & $0.0159(4)$ \\
\hline $\mathrm{C} 9$ & $0.26150(16)$ & $0.53619(10)$ & $0.6128(2)$ & $0.0148(4)$ \\
\hline C10 & $0.30384(17)$ & $0.52836(11)$ & $0.7516(2)$ & $0.0180(4)$ \\
\hline $\mathrm{H} 10$ & 0.258652 & 0.517502 & 0.799166 & $0.022^{*}$ \\
\hline C11 & $0.40906(17)$ & $0.53602(11)$ & $0.8205(2)$ & $0.0198(4)$ \\
\hline H11 & 0.435202 & 0.531886 & 0.914289 & $0.024^{\star}$ \\
\hline C12 & $0.47624(17)$ & $0.54979(12)$ & $0.7518(2)$ & $0.0203(5)$ \\
\hline C13 & $0.43954(17)$ & $0.55525(12)$ & $0.6160(2)$ & $0.0192(4)$ \\
\hline H13 & 0.486882 & 0.562684 & 0.569892 & $0.023^{*}$ \\
\hline C14 & $-0.21906(16)$ & $0.65046(12)$ & $0.3047(2)$ & $0.0182(4)$ \\
\hline H14 & -0.284811 & 0.658750 & 0.238603 & $0.022^{*}$ \\
\hline C15 & $-0.21532(16)$ & $0.65594(11)$ & $0.4412(2)$ & $0.0167(4)$ \\
\hline C16 & $-0.13137(16)$ & $0.63308(10)$ & $0.5511(2)$ & $0.0136(4)$ \\
\hline C17 & $-0.14311(17)$ & $0.63510(12)$ & $0.6769(2)$ & $0.0178(4)$ \\
\hline H17 & -0.088962 & 0.618149 & 0.751531 & $0.021 *$ \\
\hline C18 & $-0.23074(18)$ & $0.66091(12)$ & $0.6945(2)$ & $0.0208(5)$ \\
\hline $\mathrm{H} 18$ & -0.236272 & 0.662149 & 0.780373 & $0.025^{\star}$ \\
\hline C19 & $-0.31137(17)$ & $0.68524(12)$ & $0.5857(2)$ & $0.0208(5)$ \\
\hline $\mathrm{C} 20$ & $-0.30457(18)$ & $0.68177(13)$ & $0.4608(2)$ & $0.0228(5)$ \\
\hline $\mathrm{H} 20$ & -0.360955 & 0.697033 & 0.386853 & $0.027^{*}$ \\
\hline C21 & $0.15556(16)$ & $0.76880(11)$ & $0.3534(2)$ & $0.0143(4)$ \\
\hline
\end{tabular}

Table 2 (continued)

\begin{tabular}{lrrrr}
\hline Atom & $\boldsymbol{x}$ & $\boldsymbol{y}$ & $\boldsymbol{z}$ & $\boldsymbol{U}_{\text {iso }} \boldsymbol{U}_{\text {eq }}$ \\
\hline H21 & 0.152702 & 0.805744 & 0.298139 & $0.017^{\star}$ \\
C22 & $0.23772(16)$ & $0.76818(11)$ & $0.4823(2)$ & $0.0141(4)$ \\
C23 & $0.24552(16)$ & $0.71832(11)$ & $0.5779(2)$ & $0.0144(4)$ \\
C24 & $0.33087(17)$ & $0.72199(11)$ & $0.6986(2)$ & $0.0169(4)$ \\
H24 & 0.340138 & 0.687993 & 0.762316 & $0.020^{*}$ \\
C25 & $0.40041(16)$ & $0.77319(12)$ & $0.7260(2)$ & $0.0181(4)$ \\
H25 & 0.455684 & 0.775017 & 0.808781 & $0.022^{*}$ \\
C26 & $0.38976(16)$ & $0.82262(11)$ & $0.6320(2)$ & $0.0174(4)$ \\
C27 & $0.31042(16)$ & $0.82021(11)$ & $0.5118(2)$ & $0.0159(4)$ \\
H27 & 0.304467 & 0.853806 & 0.448093 & $0.019^{*}$ \\
\hline
\end{tabular}

\section{Comment}

The structural chemistry of lanthanide complexes is diverse as they can often display various coordination numbers and flexible coordination geometries. A stand-out potential application for lanthanide complexes relates to their luminescent characteristics owing to their sharp emission bands, colour tuneability and long-lived emission states [7], therefore making them potential materials as organic light-emitting devices (OLED's). Tripodal lanthanide complexes have been well characterized since the 1990's [8] and as part of an on-going study investigating related tripodal lanthanide(III) complexes [9], the crystal and molecular structures of the title neodymium(III) complex is described.

The molecular structure is shown in the figure $(70 \%$ displacement ellipsoids) and comprises a heptadentate tris $\{[5$-chlorosalicylidene)amino]ethyl $\} a m i n e)$ trianion which coordinates via the three phenolate-oxygen, three iminenitrogen and tertiary amine-nitrogen atoms. The eighth position is occupied by the aqua ligand. The ensuing $\mathrm{N}_{4} \mathrm{O}_{4}$ donor set defines a square anti-prism with one face defined by the 01, 04w, N1 and N2 atoms, while the other is defined by the O2, O3, N3 and N4 atoms. Systematic variations are noted in the bond lengths with the $\mathrm{Nd}-\mathrm{O}$ bonds being uniformly shorter than the $\mathrm{Nd}-\mathrm{N}$ bonds. Within each class of bond, the $\mathrm{Nd}-\mathrm{O}$ (phenoxide) bonds [2.2850(15)-2.3834(14) $\AA$ ] are shorter than the $\mathrm{Nd}-\mathrm{O}$ (aqua) bond [2.4916(15) $\AA$ ]. In the same way, the $\mathrm{Nd}-\mathrm{N}$ (imine) bonds [2.5829(18)-2.6882(17) $\AA]$ are shorter than the Nd-N(amine) bond [2.7335(17) $\AA]$.

The most closely related structure in the literature is that of the cerium(III) derivative, isolated as its hemi-methanol solvate. As would be expected, the mode of coordination of the ligand and distorted coordination geometry mimics that found in the title complex [10]. In addition, there are other examples with a slightly modified ligand [11].

In the crystal, water- $\mathrm{O}-\mathrm{H} \cdots \mathrm{O}$ (phenoxide) hydrogen bonding is apparent $\left[04 \mathrm{w}-\mathrm{H} 1 \mathrm{w} \cdots O 1^{\mathrm{i}}: \mathrm{H} 1 \mathrm{w} \cdots \mathrm{O}^{\mathrm{i}}=\right.$ 1.84(2) $\AA, \quad 04 \mathrm{w} \cdots O 1^{\mathrm{i}}=2.647(2) \AA$ with angle at $\mathrm{H} 1 \mathrm{w}=$ $162(3)^{\circ}$ and $\mathrm{O} 4 \mathrm{w}-\mathrm{H} 2 \mathrm{w} \cdots \mathrm{O} 2^{\mathrm{ii}}: \quad \mathrm{H} 2 \mathrm{w} \cdots \mathrm{O} 2^{\mathrm{i}}=1.941(19) \AA$, 
$\mathrm{O} 4 \mathrm{w} \cdots \mathrm{O} 2^{\mathrm{ii}}=2.723(2) \AA$ with angle at $\mathrm{H} 2 \mathrm{w}=156(2)^{\circ}$ for symmetry operation i: $-x, 1-y, 1-z]$. Each hydrogen atom of one water molecule hydrogen bonds to one of a pair of adjacent phenoxide atoms of another molecule to close a sixmembered $\{\cdots \mathrm{HOH} \cdots \mathrm{ONdO}\}$ ring. As these hydrogen bonds occur across a centre of inversion, a dimeric supramolecular aggregate ensues.

Acknowledgements: Sunway University is thanked for support of crystal engineering studies (Grant No. INT-FST-RCCM2016-01).

\section{References}

1. Bruker. APEX2 and SAINT. Bruker AXS Inc., Madison, WI, USA (2008).

2. Sheldrick, G. M.: SADABS. University of Göttingen, Germany (1996).

3. Sheldrick, G. M.: A short history of SHELX. Acta Crystallogr. A64 (2008) 112-122.

4. Sheldrick, G. M.: Crystal structure refinement with SHELXL. Acta Crystallogr. C71 (2015) 3-8.
5. Farrugia, L. J.: WinGX and ORTEP for Windows: an update. J. Appl. Crystallogr. 45 (2012) 849-854.

6. Kanesato, M.; Ngassapa, F. N.; Yokoyama, T.: Crystal structure of tris(((5-chlorosalicylidene)amino)ethyl)amine. Anal. Sci. 16 (2000) 781-782.

7. Bünzli, J. G.: On the design of highly luminescent lanthanide complexes. Coord. Chem. Rev. 293-294 (2015) 19-47.

8. Liu, S.; Gelmini, L.; Rettig, S. J.; Thompson, R. C.; Orvig, C.: Synthesis and characterization of lanthanide $[\operatorname{Ln}(\mathrm{L})]_{2} \mathrm{com}$ plexes of $\mathrm{N}_{4} \mathrm{O}_{3}$ amine phenol ligands with phenolate oxygen bridges: evidence for very weak magnetic exchange between lanthanide ions. J. Am. Chem. Soc. 114 (1992) 6081-6087.

9. Lee, S. M.; Lo, K. M.; Tan, S. L.; Tiekink, E. R. T.: (Tris\{2$\{5$-chloro-2-oxidobenzylidene- $\kappa N$ ] ethyl\}amine- $\kappa N$ ) ytterbium(III): crystal structure and Hirshfeld surface analysis. Acta Crystallogr. E72 (2016) 1390-1395.

10. Sang, Y.-L.; Lin, X.-S.; Li, X.-C.; Liu, Y.-H.; Zhang, X.-H.: Synthesis, crystal structure, and antibacterial activity of a mononuclear cerium(III) complex derived from tris(2-(5chlorosalicylidene)amino)ethyl)amine. Inorg. Nano-Met. Chem. 47 (2017) 86-90.

11. Bernhardt, P. V.; Flanagan, B. M.; Riley, M. J.: Rapid communication: completion of the isomorphous $\operatorname{Ln}($ trensal) series. Aust. J. Chem. 54 (2001) 229-232. 